\title{
Hommage
}

\section{Michel Sebillotte (1934-2010)}

Michel Sebillote, qui vient de nous quitter, a fortement marqué de son empreinte l'évolution récente de l'agronomie française au cours des nombreuses années durant lesquelles il a enseigné et cherché, à la chaire d'agronomie de l'Institut national agronomique Paris-Grignon (INA puis INA $P-G)$, puis comme directeur scientifique de l'Institut national de la recherche agronomique (Inra). Sa passion pour cette discipline l'a conduit à s'intéresser aux travaux de nombreux chercheurs et enseignants chercheurs, sous tous les cieux du monde.

Afin de lui rendre hommage, la revue Cabiers A gricultures a demandé à quelques-uns des éminents représentants de l'enseignement et de la recherche agronomique qui l'ont bien connu d'évoquer les aspects qui les ont le plus frappés de la personnalité de cet homme d'exception.

Ce sont ces textes qui figurent ci-dessous. Tous ceux qui voudraient mieux connaître l'cuvre de Michel Sebillotte dans sa (presque) totalité, pourront se référer par ailleurs au texte qu'il avait lui-même rédigé en 2001 et que l'Inra a publié dans la collection "Bilans et Prospectives », intitulés: "Apprendre, chercher, Innover. Les parcours d'un agronome».

$\begin{array}{ll}\text { Jean-Pascal Pichot } & \text { Didier Picard } \\ \text { Rédacteur en chef } & \text { Rédacteur en chef délégué }\end{array}$

$80 \sec 603$

Hubert Manichon
Ancien professeur d'agronomie à l'INA P-G
Ancien directeur scientifique du Cirad

J'ai fait la connaissance de Michel au printemps 1965, grâce à Stéphane Hénin. Celui-ci venait de m’interroger pour l'examen oral d'agriculture de deuxième année (j'appartenais à la dernière promotion de l'INA qui avait eu l'immense chance de bénéficier de l'enseignement de cette personnalité hors pair) et cette épreuve s'était plutôt bien passée. Appelant Michel qui était dans le bureau voisin, il lui dit: «Sebillotte! Vous devrez prendre Manichon dans votre équipe... ». Un tel parrainage était élogieux mais, vis-à-vis de Michel - et de ce que beaucoup pourraient appeler son esprit de contradiction - ce n'était pas forcément une bonne introduction! Eh bien si: nous nous sommes rapidement reconnus en connivence pour avoir eu tous deux des pères agriculteurs passionnés de leur métier et qui avaient su être pionniers dans leurs domaines. De cette ascendance, Michel avait pris goût pour les combats réputés impossibles...

À l'époque, la situation de la chaire d'agriculture ${ }^{1}$ à l'INA n'était pas fameuse: retour de Stéphane Hénin vers l'Inra pour diriger le Département d'agronomie, départ de Jean-Pierre Deffontaines à l'Inra pour la création du SEI (ancêtre du Département SAD de l'Inra $)^{2}$. Michel venait d'être nommé maître-assistant ${ }^{3}$ et responsable de la chaire. Grâce à l'appui de Roger Blais, directeur de l'INA, il avait obtenu la possibilité de recruter des assistants pour faire face aux besoins de l'enseignement, je fus l'un d'eux.

Dans l'état d'esprit de l'époque il était inconcevable, avec le grade de maître-assistant, de faire cours en amphi; nous eûmes donc recours à des conférenciers (Gérard Monnier et Jean Hébert, tous deux chercheurs) pour remplacer Stéphane Hénin. Je crois pouvoir dire que pour Michel, au-delà de l'estime et de l'amitié qu'il éprouvait pour eux, cette situation ne pouvait qu'être transitoire...

L'amphithéâtre étant temporairement hors de portée, il fallait mener le combat et relever les défis ailleurs. C'est-à-dire partout: enseignement à tous niveaux, formation continue, coopération internationale, insertion avec les acteurs du développement et les agriculteurs, avec les décideurs économiques... et bien sûr recherche. Ainsi, les réflexions conduites pour concevoir un enseignement d'agronomie pour les stages en exploitation agricole des étudiants de l'INA trouvaient leur écho dans la formation des techniciens agricoles des chambres d'agriculture: l'observation du profil cultural, l'analyse des pratiques agricoles, l'analyse de la diversité des exploitations agricoles dans une région, la comparaison des filières et des régions... étaient d'abord des lieux d'initiation à l'analyse systémique du réel, à haute valeur formatrice pour les étudiants, avant de devenir des domaines de recherche.

${ }^{1}$ À l'époque, « agriculture » et non pas « agronomie ». Michel, on le sait, a beaucoup œuvré pour donner du sens au second terme.

${ }^{2}$ SEI : Service d'expérimentation et d'information, créé au sein de l'Inra en 1964, et succédant au SARV (Service d'adaptation de la recherche à la vulgarisation) créé auparavant en 1959. SAD: Département Systèmes agraires et développement créé en 1979. La fréquence des réformes institutionnelles montre la difficulté d'un grand sujet (les relations entre recherche et développement, l'interdisciplinarité) qui a passionné Michel tout au long de sa vie.

${ }^{3}$ Grade correspondant à l'actuel maître de conférence. 


\section{Hommage}

J'en témoigne: pendant au moins 20 ans, c'est la fonction de formation, au sens large, qui a été première. Michel recherchait, et trouvait le plus souvent, dans des discussions d'une très grande liberté avec les étudiants, la contestation et la validation des idées nouvelles. Parallèlement, on ne peut faire le décompte des heures de réflexion, de discussion, d'affrontements intellectuels, toujours animés par Michel au sein de l'équipe d'enseignants qui s'était progressivement constituée et enrichie. Chaque lecture nouvelle, chaque tribune nouvelle étaient des occasions et des moyens de développer la réflexion et de la mettre à l'épreuve ${ }^{4}$. Les publications de Michel les plus importantes de cette époque (agronomie et agriculture, itinéraires techniques et évolution de la pensée agronomique, systèmes de culture...) sont issues de cette dynamique.

Et il fallait aussi, bien sûr, s'inscrire dans le paysage de la recherche, et contribuer au développement des connaissances et des méthodes. Or, Stéphane Hénin n'avait pas mis en place, lorsqu'il était professeur d'agriculture à l'INA, de réels moyens de recherche au sein de sa chaire d'enseignement ${ }^{5}$. Il estimait, je crois, que la recherche devait être faite avec son propre rythme et son propre champ, qui ne sont pas ceux de l'enseignement. Au moment où Michel a pris cette succession difficile, la situation à l'INA était ainsi très originale: contrairement à la plupart des autres écoles, la chaire de l'INA ne disposait ni de laboratoires assez étoffés, ni de domaines expérimentaux liés à l'Inra. Ce fut pour nous une chance: il fallut innover, oser... pour produire scientifiquement. Très concrètement, on choisit de promouvoir l'enquête comme moyen d'investigation dans le réel, puisque le dispositif «Fischérien ${ }^{6} »$ n'était pas disponible. Or, ce dernier dispositif, plus traditionnel, était orienté en agronomie presque exclusivement vers l'analyse statistique d'effets de traitements expérimentaux, mesurés au travers du rendement des cultures. Autrement dit vers l'analyse de l'effet de la technique (fertilisation, travail du sol, variété...) sur le rendement... Schématiquement, on substitua à cette relation statistique «aveugle » des tentatives de compréhension et d'explication de la variabilité des résultats finaux, en étudiant séparément (autant que faire se peut) une première relation de type «technique x état du milieu de culture », et une seconde de type « état du milieu x rendement». Cela est loin d'être anecdotique ou sans conséquence sur les dispositifs d'enquête et les protocoles, qu'il a fallu tester, codifier, mais aussi instrumentaliser! En effet, nous étions partis d'une recherche à base de «bouts de ficelle », sans moyens techniques impressionnants, qu'il a bien fallu enrichir en métrologie et méthodes d'analyse de données pour rendre les résultats opposables, et... académiquement publiables! Cela n'aurait, par ailleurs, pas été possible sans l'amicale attention d'un Guy Lefort et d'une Camille Duby, mathématiciens compagnons de route d'agronomes ne suivant pas les chemins de la tradition, et capables d'aide et d'inventivité méthodologique au service de la mesure et de la validation des faits issus du terrain.

À la fin des années 1970, une session du «Cycle supérieur d'agronomie » (entité de formation continue créée à l'initiative de Michel) consacrée à « Enquêtes et expérimentations: valeurs respectives pour l'analyse des systèmes agricoles » offrit, au milieu d'autres contributions, une synthèse de presque 15 années de travaux de recherche engagés à la chaire d'agriculture de l'INA. Le public relevait des institutions de recherche (avec une participation remarquée de la recherche en milieu tropical), des instituts techniques agricoles et des institutions de formation. Il ne reste malheureusement pas de document publié de cette opération. Mais les idées d'alors ont contribué à l'enrichissement des thématiques scientifiques du Département Agronomie de l'Inra, à la problématique du Département Systèmes agraires et développement de l’Inra, ainsi qu'à la conception des opérations de « relance agronomique » du début des années 1980. Un sujet qui, semble-t-il, redevient d'actualité...

$80 \operatorname{soc} 00$

$$
\begin{gathered}
\text { Alain Capillon } \\
\text { Professeur à Montpellier SupAgro }
\end{gathered}
$$

Comme pour beaucoup d'autres, c'est Michel Sebillotte qui m'a donné l'envie de faire de l'agronomie. Après m'avoir attiré dans sa section de spécialisation, il m’a accueilli dans son équipe d'enseignants-chercheurs. J'ai eu l'immense honneur de lui succéder à la tête des agronomes de l'INA P-G quand il a pris ses fonctions à l'Inra. Parmi ses nombreuses activités, je retiendrai ici, celles concernant la formation des agronomes.

Michel Sebillotte a conçu et forgé non seulement un enseignement mais une doctrine d'agronomie dans la continuité des apports de Stéphane Hénin. Il a défini, fait vivre et enseigné des concepts nouveaux, fondateurs d'une agronomie moderne «à la française »: systèmes de culture, itinéraire technique, fonctionnement et trajectoires d'évolution des exploitations agricoles. Il a su intégrer des contributions de disciplines de sciences sociales et humaines pour définir le concept de modèle général et de modèle d'action, si utiles pour comprendre, interpréter puis guider les décisions des agriculteurs. Ces concepts essentiels à la discipline donnent les bases d'une agronomie systémique désormais admise et établie.

${ }^{4}$ Je me reconnais dans la manière dont Jean Boiffin en parle dans son propre témoignage.

${ }^{5}$ Contrairement à ce que la chaire de zootechnie avait réalisé à la même époque sous l'impulsion de J. Delage et J. Coléou.

${ }^{6}$ Avec toutes les « garanties » de traitement statistique des données expérimentales qu'il devait offrir. 


\section{Hommage}

Tous ces apports notables sont le fait d'un fils d'agriculteur soucieux de la confrontation au terrain qu'il a fortement contribué à instrumenter mais aussi de contacts et de dialogues avec les acteurs du développement agricole qu'ils soient agriculteurs, techniciens ou gestionnaires de filières. Il nous a appris à être interrogés par le terrain qui n’est plus vu comme une simple application de la théorie mais aussi et surtout comme lieu de réflexion, voire de création. Celui-ci ne se limite pas à la parcelle; pour Michel, pas de terrain sans dialogue avec l'agriculteur. Ainsi conçu, c'est bien un lieu de formation. Dans la conception des enseignements d'initiation à l'agronomie, nous faisons, encore aujourd'hui appel à ses démarches inductives et mettons en œuvre sa philosophie de la confrontation au terrain et aux acteurs.

Dans tous ces domaines, il a été pionnier, pratiquant la «philosophie du non » et doté d'un regard fondé sur l'approche critique des sciences baignée par l'épistémologie bachelardienne. Formateur d'ingénieurs et d'hommes d'action, il a su leur donner le goût de la réflexion scientifique et du retour critique en initiant un cours d'épistémologie à l'Agro de Paris.

C'est sans doute dans ces deux préoccupations qu'il faut voir le succès de Michel auprès des étudiants mais aussi des chercheurs ingénieurs, techniciens et agriculteurs : un souci constant, d'une part, de confronter théorie et constat sur le réel, d'assister les apprentis agronomes dans la douloureuse pratique de l'induction, et d'autre part, de développer une réflexion critique sur le caractère scientifique de ces réflexions et interprétations. C'est ainsi que dans le domaine de la recherche, il a milité pour faire reconnaitre le caractère heuristique et scientifique de la démarche par enquêtes, qu'elle se déroule en champs d'essais, en parcelles ou en exploitations, et qu'il excellait dans la manière de transformer les problèmes perçus en problématique et en projet de recherche.

Michel Sebillotte a été précurseur, leader et premier de cordée pour ceux qui l'ont suivi, ce qui était parfois difficile du fait de sa rapidité d'action, de ses intuitions nombreuses et de son tempérament de battant! Dans beaucoup de domaines, dont certains débordent largement de l'agronomie, comme le développement régional ou la prospective, sa pensée est visionnaire. Je voudrais terminer ce trop court et humble hommage en citant un paragraphe tiré de la page 19 de son ouvrage «Fertilité et systèmes de production »:

\section{"C'est tout particulièrement le cas des scientifiques dont on sait combien ils participent fortement aujourd'bui aux représentations sociales dans un monde baigné de scientisme et donc toujours prêt à valoriser fortement leurs déclarations. Or, si le scientifique s'insurge face aux explications trop grossières lorsqu'il s'agit de son champ d'activité, il perd souvent une grande partie de son esprit critique dans les domaines qui sortent de sa compétence; il discourt avec l'évidence du bon sens et, du coup, devient prisonnier des représentations sociales dominantes tout en les entretenant. Il se met au service de causes, réagit à partir de ses propres valorisations, se libère ainsi, peut-être, des contraintes qui enserrent sa pratique dans son propre champ de travail. »}

Ce texte publié en 1989 donne un judicieux éclairage aux discours actuels sur l'évolution de la planète ou sur le développement durable de certains scientifiques! Il incite, de fait, les enseignants à entraîner les futurs chercheurs ou ingénieurs à l'analyse critique, épistémologique de textes prétendument scientifiques ou émanant de scientifiques comme Michel nous l'avait fait pratiquer.

\section{$80 \operatorname{sos} 03$}

Jean Boiffin
Directeur de recherche Inra
Président de l'Association française d'agronomie

Il y aura beaucoup à faire pour inventorier et mettre en valeur l'immense apport de Michel Sebillotte aux agronomies: à l'agronomie discipline scientifique et technologique, qui lui doit d'être reconnue comme telle; à l'agronomie au sens large, qui a grandement bénéficié de sa réflexion sur la recherche finalisée, et de ses explorations en prospective et en développement régional. Cet apport est jalonné d'écrits et de réalisations qui en sont les témoins durables. Mais ceux qui, comme moi, l'ont côtoyé de près, doivent aussi témoigner de ce qui risque plus d'être oublié et méconnu: l'attraction et la capacité de mobilisation qu'il a exercées auprès des centaines d'étudiants qu'il a formés à l'agronomie, et des personnes encore plus nombreuses qu'il a entraînées dans ses entreprises. Ce rayonnement doit beaucoup à une personnalité d'exception dont l'intelligence, l'énergie, la puissance de travail, la verve, l'insolence maitrisée, resteront gravées dans les mémoires... Michel n’aurait pas détesté qu'on le rappelle, mais il n’aurait pas aimé qu'on en reste là.

Pourquoi ai-je choisi de suivre Michel, d'abord en m’inscrivant dans la spécialisation de la troisième année dont il était responsable, puis en me portant candidat à un poste d'assistant au sein de la « chaire d'agriculture » de l'INA? Et ce, alors même qu'en pleine année 1968, il se présentait aux étudiants en arborant costume et nœud papillon, et de façon plus que bourrue... Parce que dès ses premiers mots, il avait exprimé avec force et justesse un projet didactique qui entrait en résonance avec les aspirations d'un étudiant insatisfait de l'abîme qu'il mesurait entre un savoir encyclopédique et l'univers de l'action, de l'agriculteur, 


\section{Hommage}

de l'ingénieur qu'il était censé devenir. Michel nous proposait de construire puis mettre en œuvre une démarche de pensée qui nous recréait comme acteur et sujet de notre propre formation et qui faisait le lien entre le savoir et l'action. À son contact, et grâce à la richesse des exercices d'observation, de diagnostic, de synthèse, de prescription, de lectures épistémologiques, auxquels il nous a confrontés en tant qu'étudiants ou jeunes enseignants, nous avons eu le sentiment non seulement d'apprendre puis de transmettre un vrai métier, mais aussi de participer à son développement.

À travers ce métier, nous entrions en interaction confiante, pleine d'estime réciproque, aussi bien avec les praticiens qu'avec les grandes personnalités scientifiques et agronomiques de l'époque - Stéphane Hénin, Gérard Monnier, Jean-Pierre Deffontaines, Raymond Gras, pour ne citer que le père et les frères spirituels de Michel. Du désarroi intellectuel à la fierté: voilà le parcours au long duquel nous a guidés Michel. Une fierté sans autosatisfaction, car durement et jamais définitivement acquise: Michel se, et nous, soumettait à une exigence intellectuelle intransigeante, se traduisant par un terrible esprit critique, mais aussi par une impitoyable exigence constructive : il détruisait tout de fond en comble, mais s'obligeait et nous obligeait à ne jamais en rester là. Plus profondément encore, il nous a appris et transmis le goût de la maïeutique, cette marque de fabrique de la famille Hénin: Michel a été un formidable animateur d'équipe, capable de rendre créatif n'importe quel groupe de travail. Nous gardons tous le souvenir de séances mémorables où, sur un tableau noir ou une nappe de bistrot en papier, s'élaborait le graphe de relations qui allait structurer un enseignement, une thèse, un projet d'article. Après les éprouvantes critiques qui réduisaient à néant nos réflexions préliminaires, venait le temps de la construction, de la mise en ordre, de l'enrichissement mutuel, de la valorisation méticuleuse des apports de chacun, et pour finir de la jubilation d'avoir contribué à une création collective.

J'ai eu la chance de bénéficier de ces interactions fructueuses en travaillant directement avec lui, à l'INA puis plus tard à l'Inra. Mais son rayonnement s'est exercé dans de très nombreux autres cadres. La diversité des sujets qu'il a explorés et des communautés avec lesquelles il entrait en contact donne à réfléchir. S'il ne s'y est jamais perdu, c'est sans doute en raison de son exigence d'ancrage dans le concret: les exercices spéculatifs auxquels il se livrait sans retenue avaient toujours pour point de départ et de retour l'observation, les données, les faits, les actes. Même si Michel nous donnait parfois le tournis en s'attaquant à des ascensions vertigineuses dans des domaines imprévisibles, il y avait toujours moyen de partager avec lui la passion de l'observation et de l'interprétation du réel. De ce fait même, ses explorations intellectuelles ont toujours été accompagnées avec plaisir par un grand nombre de personnes, représentant des gammes très variées de métiers, du praticien au théoricien.

Tous les agronomes d'aujourd'hui, quel que soit leur parcours, et même s'ils ne l'ont pas directement connu, doivent à Michel Sebillotte une part de la fierté et du plaisir qu'ils ont à exercer leur métier et de la sympathie dont il jouit auprès d'un large public. En retour, nous devons à Michel de faire fructifier ce trésor, de continuer à l'enrichir, et de continuer à le transmettre.

8c) $80 \operatorname{sos}$

Marianne Cerf

Directeur de recherche Inra

Michel Sebillotte a bien sûr d'abord été un enseignant, ou peut-être devrais-je dire un formateur. Il mettait toute sa conviction et sa force argumentative - ce qui pouvait sembler parfois aux étudiants de l'INA P-G de la hargne - pour les interpeller sur ce qu'est la science, en affirmant que l'agronomie est une science, même si ses objets et ses méthodes semblaient assez peu scientifiques aux étudiants de première année après les deux années de classes préparatoires. Au-delà de sa volonté de bousculer les certitudes des étudiants, il cherchait surtout à leur faire saisir comment une science comme l'agronomie est une science pour et de l'action, et comment il fallait la construire pour permettre de penser et d'accompagner l'action des agriculteurs. Ce n'était pas qu'un discours destiné aux étudiants. C'était aussi une pratique, qui s'est traduite entre autres par son investissement important auprès des acteurs du développement agricole. Beaucoup d'agronomes qui exercent leur métier au sein des structures de R\&D et de conseil en France ont connu Michel lors de l'opération de formation continue et de développement appelée « relance agronomique » dans les années 1980, ou lors des opérations « Ferti-mieux » et « Irri-mieux » dans les années 1990. Ils évoquent souvent son apport méthodologique incontestable, ainsi que son souci permanent que ces opérations conduisent réellement à un apprentissage des bases agronomiques qu'il considérait comme utiles à l'action des acteurs du développement auprès des agriculteurs. C'était aussi cette agronomie «science pour et de l'action » qu'il a développée à l'occasion des recherches qu'il dirigeait au Département SAD de l'Inra au début des années 1980. J'évoquerai ici un point qui me parait emblématique de cette ambition: les programmes de recherches qu'il a dirigés en vue de modéliser les processus de décision des agriculteurs. Il va alors puiser les ressources intellectuelles et méthodologiques pour saisir ces décisions dans diverses disciplines. Cette curiosité pour d'autres disciplines a toujours été un trait marquant de sa démarche, et les années 1980 voient arriver dans le laboratoire qu'il dirigeait des chercheurs en gestion, en économie, en psychologie sociale. Cette curiosité à l'égard d'autres disciplines est à relier, me semblet-il, à la façon dont Michel construisait une question de recherche. N'est-ce pas dans une intelligence des situations avant tout, et 


\section{Hommage}

non dans la volonté unique de tester des hypothèses déduites de cadres théoriques, qu'il a su développer une agronomie comme science de et pour l'action? Ainsi, n'est-ce pas fort de son expérience propre d'agriculteur, de la place qu'il a toujours donnée au « tour de plaine », qu'il a eu l'intuition que l'observation au plus près de ce que font les agriculteurs était nécessaire à une meilleure compréhension de ce qui oriente l'action, et qu'il a mobilisé alors l'ergonomie, qui met au cœur de son programme de recherche l'observation de l'activité des opérateurs?

En investissant le champ de l'ergonomie, Michel a ainsi vu la possibilité d'entrer dans la logique d'action des agriculteurs en situation. L'enjeu était de reconnaître la radicalité de la différence entre une logique de compréhension d'un système sociotechnique et une logique d'utilisation de ce système, différence énoncée dès le début des années 1980 par J.-F. Richard, alors professeur à l'Université Paris 8, qu'il a su intéresser à l'agriculture. Plus précisément, Michel souhaitait, grâce à cet emprunt, comprendre comment se mêlent connaissances issues d'une confrontation à la résistance du réel (apprentissage " sur le tas ») et connaissances venant des multiples prescripteurs qui gravitent autour des agriculteurs. La didactique professionnelle, qui peut aujourd'hui aider à traiter cette question, n'était à l'époque que balbutiante... ainsi la question formulée à la fin des années 1980 commence seulement maintenant à pouvoir être pleinement saisie dans les dimensions que Michel aurait aimé voir aborder. À l'époque, le travail est resté ancré sur la logique d'action et le rapport qui s'instaure entre diagnostic d'une situation et connaissances des agriculteurs sur l'action.

C'est autour de ce mouvement entre «terrain », mobilisation de ressources théoriques, construction d'une problématique et d'une intelligibilité des situations que se sont poursuivis mes échanges avec Michel, une fois son engagement dans le suivi direct des travaux de recherche limité par ses autres charges. Nous n'étions pas toujours d'accord sur la façon d'aborder la question du rapport entre connaissances et action, et en particulier dès que cela touchait à l'agronomie comme science pour et de l'action. Dès lors que l'agronome construit l'agronomie comme une science pour l'action, comment choisit-il de se représenter les rapports entre connaissances et action? Comment mobilise-t-il les différentes théories développées par les sciences humaines et sociales pour traiter de ce rapport? Comment explore-t-il, en situation, la façon dont ce rapport s'instaure à la fois pour comprendre la logique d'organisation des opérations culturales et pour engager un dialogue fructueux entre l'agronome prescripteur et celui qui réalise ces opérations? Autant de questions que j'aurais aimé pouvoir encore discuter avec Michel. Nous avons perdu un interlocuteur exigeant, disposant d'une expérience mêlant production scientifique et implication forte auprès des agriculteurs et des acteurs du développement, et souhaitant mettre à distance, dans une réflexion à caractère épistémologique, cette expérience. Il nous reste néanmoins ses écrits et, pour ceux qui ont eu la chance de le côtoyer, le souvenir très présent de ce qu'il était dans l'action.

\section{$\operatorname{sos} \cos 3$}

François Papy

Ancien directeur de recherche Inra

\section{Michel derrière Sebillotte}

Sebillotte examinait un profil cultural, lorsque je l'ai rencontré pour la première fois. C'était au cours de l'hiver 1970, à Montluel (Ain), chez les frères Lassus où il avait mis en place, en 1963, un essai de rotation culturale. J'y conduisais un voyage d'étude de la première promotion de l'Enita de Dijon. Cherchant à impressionner les élèves (et moi aussi sans doute), il avait dit: «Bof! sept ans ce n'est rien pour un essai de longue durée. On n'a pas le temps de se retourner que c'est déjà passé! »". Un an plus tard, je devais revoir Sebillotte dans son bureau de Paris. C'est qu'entre-temps Roger Blais, directeur de l'Agro, m'avait proposé de partir en détachement pour monter l'enseignement d'agronomie à l'Institut agronomique et vétérinaire Hassan II de Rabat qui venait d'être créé. J'hésitais. Je ne voulais pas y partir sans appui. Je venais chercher de l'aide auprès de Sebillotte. Ce jour-là nous avons conclu une affaire qui allait durer neuf ans. C'est ainsi qu'il viendra en mission, deux fois l'an les premières années, épauler l'équipe qui se monte à Rabat, puis, plus tard une seconde à Meknès ${ }^{8}$; il enverra tous les ans un ou deux des élèves sortant de sa section au titre du volontariat pour le service actif (VSNA); et, le moment venu, il prendra à Paris les élèves marocains qui, en fin de cycle de formation, se spécialisent en agronomie.

Hébergé à la maison, emmené sur le terrain aux quatre coins du pays avec toute l'équipe ${ }^{9}$, très vite, Sebillotte est devenu Michel. Au cours des tours de Maroc, Sebillotte nous initie à la recherche en agronomie: aux enquêtes dans les champs et auprès des

${ }^{7}$ L'essai durera 21 ans!

${ }^{8}$ Sous la houlette de Jean Fenech.

${ }^{9}$ Constituée de Philippe Jouve, Myriam Henrion, Jean Fenech et François Lelièvre, sans parler des nombreux VSNA qui se sont succédé. 


\section{Hommage}

agriculteurs pour faire des diagnostics sur les effets des pratiques et les possibilités de faire autrement. C'est toujours Sebillotte qui anime les discussions et nous nourrit de littérature agronomique. Mais c'est Michel qui partage avec nous la beauté des paysages, goûte à la finesse de la cuisine marocaine, s'enquiert dans les courriers des nouvelles des uns et des autres. Je me souviendrai toujours, dans un boui-boui de Oualidia, d'une discussion de Sebillotte avec Paul Pascon, le grand sociologue de l'Institut de Rabat qui fut l'âme de la pédagogie par les stages. L'un et l'autre, qui s'appréciaient mutuellement, discutaient âprement, comme deux pieds-noirs qu'ils étaient, à qui aurait raison de l'autre: un match Sebillotte-Pascon. Mais c'est Michel qui, lors de sa remise de légion d'honneur a trouvé les mots pour rendre un hommage très personnel à la mémoire de Paul Pascon, disparu entre-temps. Lorsque je suis renté en France, Sebillotte a favorisé mon détachement à l'Inra et m'a pris comme adjoint dans son unité de recherche du Département Systèmes agraires et développement qui venait d'être créé. Proche collaborateur de Sebillotte, j’ai bien connu Michel. Nous ne dirons jamais assez tout l'apport de Sebillotte à l'agronomie telle qu'elle est pratiquée en France. Nombre des idées qu'il a su conceptualiser sont maintenant devenues familières. Avec le recul nécessaire, viendra le temps de lui rendre hommage. Aujourd'hui, c'est Michel que je voudrais ne pas oublier. Un Michel pas toujours facile d'accès, masqué par la force intellectuelle de Sebillotte et - il faut bien le dire - son ego. Michel derrière Sebillotte. Michel sensible, attentionné. Fragile même.

Lorsque, quelques mois avant qu'il ne nous quitte, je suis allé le voir boulevard Massena, alors que je venais de lui dire que beaucoup d'amis s'inquiétaient de son état, Michel m'a répondu: «Tu crois? ». J'espère, maintenant, qu'il n'en doute plus.

\section{$80 \operatorname{sos} 03$}

\section{Jean-Yves Jamin \\ Chercheur au Cirad \\ Georges Serpantié}

Chargé de recherche IRD

\section{Michel Sebillotte et les Dogons: de la thèse à la falaise et de la falaise à la thèse...}

Comme beaucoup, c'est sur les bancs de l'Agro que nous avons d'abord connu Michel Sebillotte, Seb, comme on disait alors. Et toi, tu fais quoi, l'an prochain? Seb... Cette troisième année de spécialisation fut l'occasion de connaitre un peu mieux celui qui était déjà un monument de l'Agro, et qui nous avait, au cours des années précédentes, captivés, et parfois, il faut bien le dire, agacés. Mais nous en redemandions... Nous étions alors en 1979. Exploitations agricoles, typologies, systèmes de culture, étaient notre pain quotidien.

Quelques années plus tard, jeunes chercheurs fringants courant l'Afrique, l'un pour l'Irat, aujourd'hui Cirad, l'autre pour l'Orstom, aujourd'hui IRD, nous essayions d'exercer les questionnements de l'agronomie sur des situations africaines, l'un au Sénégal puis au Mali, l'autre en Côte d'Ivoire puis au Burkina Faso. Et lorsque nous avons tenté cette aventure de la thèse, nous nous sommes tournés vers le Maître. On disait le Maître en se moquant tout à la fois de lui et de nous... mais nous n'avons pas le souvenir que nous ayons ainsi désigné un autre prof. Au-delà de la dérision, il y avait le respect.

Nous avons donc invité Michel Sebillotte à venir nous rejoindre sur le terrain, pour bénéficier au mieux de son encadrement: à Paris, c'était toujours difficile, il était insaisissable, toujours pris entre des cours, des conseils scientifiques, les batailles de l'Inra et du SAD, l'Académie d'agriculture. Georges avait réussi à déjeuner une fois avec lui! Il n'a pas hésité une seconde à dire oui. Mais il y avait une condition, une seule: les Dogons! Michel voulait bien venir patauger dans les rizières de Niono, mais il voulait aller chez les Dogons. Condition sine qua non. Pas de Dogons, pas de thèses. Donnant-donnant. Bon, pas très étonnant pour le visiteur standard qui vient au Mali, de vouloir aller au pays Dogon. Cela fait partie des incontournables touristiques, et, parents, amis, tous ceux qui venaient nous rendre visite voulaient évidemment y aller. Cependant, Michel paraissait si loin de ces préoccupations touristiques que nous fûmes un peu étonnés. Mais au fond, pas fâchés de ne pas passer tout notre temps avec lui à parler agronomie, enracinement, élaboration du rendement, pilotage de l'exploitation... ou références théoriques.

Quand Michel arriva, nous comprîmes mieux: il en connaissait déjà plus sur les Dogons avant de poser le pied au Mali que nous qui étions en Afrique depuis 8 ans. Il avait tout lu sur eux, et bien sûr, par-dessus tout, les bouquins de Marcel Griaule, devenus sa Bible. Les touristes, c'étaient nous! Lui, la cosmogonie, les rites, les cérémonies, il avait tout lu et relu... mais il voulait voir. 


\section{Hommage}

Nous partîmes donc. Première étape dans un campement du côté de San. À l'époque, campement administratif, le désengagement de l'État n'était pas encore à la mode. Pas d'eau, pas d'électricité, une lampe à pétrole, et pour manger, les gargotes le long de la route. Comment allait réagir le Maitre? Le Professeur parisien? Mais il était ravi: c'était pour les Dogons...

Sur place, se posa la question du type de visite à faire: petit tour, moyen tour, grand tour? Michel n'était déjà plus tout jeune, et nous étions en juillet, autant dire qu'il faisait chaud, très chaud, et lourd aussi. A chaque pas, on dégoulinait. Mais Michel voulait tout voir, tout visiter, marcher partout. Après une discussion où chaque membre de l'équipe fut soigneusement sondé, révélant le sens démocratique très pointilleux de Michel, le consensus se fit pour le grand tour... Et nous avons donc marché. Des kilomètres de marche au sommet de la falaise, dans les rochers. Puis dans la falaise, au milieu des villages Dogons et des anfractuosités où se nichent les restes de la civilisation Telem. Et enfin, au bas de la falaise, dans les sables du Seno... où tout ce sable fait regretter les sentiers caillouteux du sommet. Nous étions tous cuits, rôtis de soleil, dégoulinant de fatigue, mais nous étions chez les Dogons, dans cette falaise mythique qu'animent quelques cascades à cette période de l'année. Le guide n'eut guère de repos: malgré la fatigue de notre petit groupe, il était soumis au feu roulant des questions de Michel. Il voulait tout savoir, tout vérifier, retrouver sur le terrain, dans ces villages, le fruit de ses lectures: il avait tout Griaule en tête, depuis les dieux jusqu'aux oignons, et nous avions souvent l'impression qu'il en savait plus que notre guide!

Puis, il fallut bien remonter la falaise. Nous étions un peu inquiets : Michel était épuisé, en nage... mais il tenait le choc: il était «Heu-reux »! Après cette ultime longue, très longue montée, vint le moment des achats: que ramener de ce pays Dogon qui puisse en symboliser l'âme? Quelle statuette? Quelle poterie? Quel jouet d'enfant? Quel outil dont on avait tant discuté de l'usage et des effets sur les «États du Milieu »? C'est à Georges qu'échut la lourde tâche du marchandage, dont l'objet était - qui s'en étonnera? - des outils agricoles. Car Michel voulait absolument payer le juste prix, ni plus, ni moins, et c'est dire s'il ne voulait être pris par nos hôtes Dogon ni pour un touriste ni pour un bienfaiteur, mais juste pour le témoin admiratif d'une société agricole aux prises avec un milieu aussi contraignant que ce plateau rocheux sahélien.

Au final, nous sommes tous repartis épuisés. Mais cette longue marche et ces presque aussi longs marchandages avaient changé nos relations avec le Maître. Il était devenu plus humain, et surtout il avait pris une autre dimension: on pouvait être un puits de sciences agronomiques, et être cultivé, s'intéresser non seulement aux mottes de terres, mais aussi aux gens. Et pas seulement à la rationalité agronomique de ces gens: mais aussi à leur culture et leurs croyances... même les plus irrationnelles.

Dès lors, nos relations ne furent plus vraiment les mêmes. Les relectures des chapitres de la thèse ne se firent plus à l'Agro, mais dans le $13^{\mathrm{e}}$ arrondissement, chez Michel, dans cet appartement qui dominait un quartier devenu presque aussi exotique que le pays Dogon dominé par sa falaise. Même si ce démarrage de doctorat ne fut qu'une étape pour Georges, puisqu'il a achevé sa thèse avec François Papy, il fut inoubliable. Pour Jean-Yves, finir la thèse ne fut pas non plus un chemin pavé de roses - ce fut long, très long, bien plus qu'un grand tour dans la falaise -, et il reste persuadé que sa thèse, c'est peut-être un peu aux Dogons qu'il la doit... 